$B B$
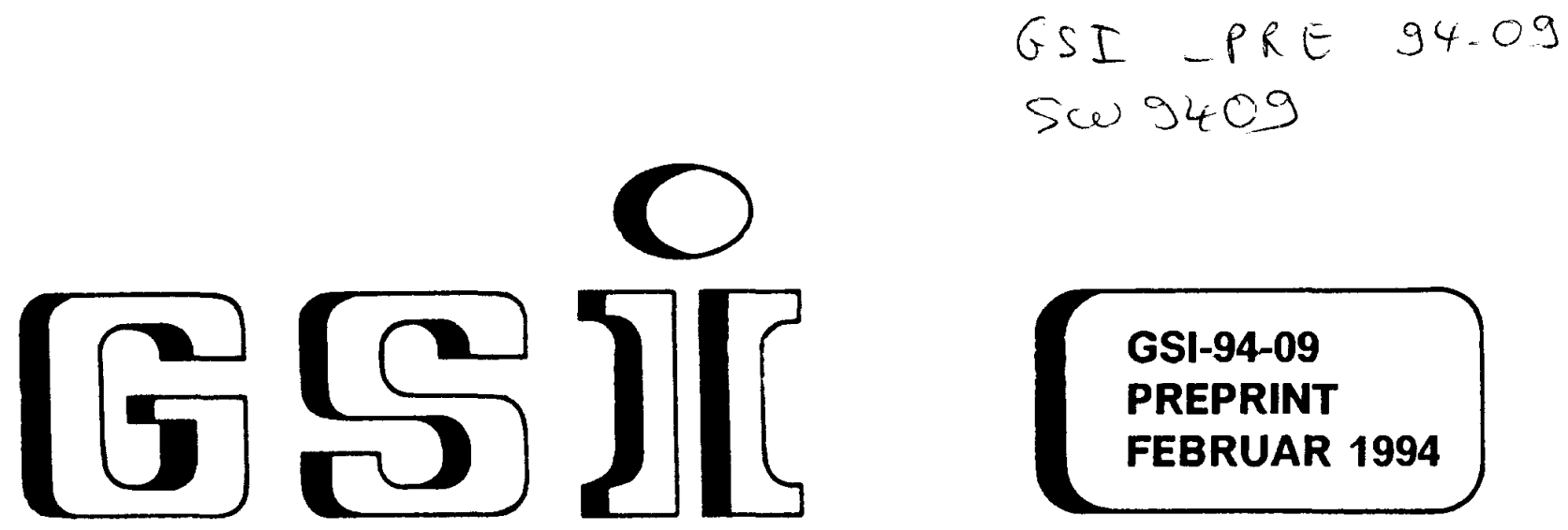

GSI-94-09 PREPRINT FEBRUAR 1994

STOCHASTIC MULTI-FLUID MODELS FOR INTERMEDIATE-ENERGY HEAVY-ION COLLISIONS

S. AYIK, Y.B. IVANOV, V.N. RUSSKIKH, W. NÖRENBERG

Gesellschaft für Schwerionenforschung $\mathrm{mbH}$ Postfach 110552 - D-64220 Darmstadt · Germany 



\title{
Stochastic Multi-Fluid Models for Intermediate-Energy Heavy-Ion Collisions $\pi$
}

S. Ayik ${ }^{a}$, Y.B. Ivanov ${ }^{b, *} \dagger$, V.N. Russkikh ${ }^{b, *}$ and W. Nörenberg ${ }^{b, c}$

a Tennessee Technological University, Cookeville, TN 98505, USA

and Joint Institute for Heavy Ion Research, Oak Ridge, TN 37891 , USA

${ }^{b}$ Gesellschaft für Schwerionenforschung (GSI), D-64220 Darmstadt, Germany

${ }^{c}$ Institut für Kernphysik, TH Darmstadt, D-64289 Darmstadt, Germany

February 2, 1994

\begin{abstract}
The relativistic Boltzmann-Langevin equation is reduced to a stochastic multi-fluid model and the corresponding transport coefficients, i.e. the interflow friction force and the interflow diffusion tensor, are extracted. The model is applied to fluctuation phenomena in the equilibration process for two counter-streaming nuclear fluids.
\end{abstract}

I This work is supported in part by US DOE grant DE-FG05-89ER40530

* Permanent address: Kurchatov Institute, Moscow 123182, Russia

$\dagger$ Alexander-von-Humboldt fellow 


\section{Introduction}

Many aspects of nuclear collisions can be described in terms of relativistic single-particle transport models based on effective quantum-field theories. In these transport models the system is represented by its one-body density matrix or its Wigner transform (Wigner function) rather than by its many-body density matrix. In the semi-classical limit the Wigner function reduces to a single-particle phasespace distribution function which is determined by a transport equation containing self-consistent mesonic mean fields and a binary collision term of the BoltzmannUehling-Uhlenbeck (BUU) type $[1,2,3,4,5,6,7,8,9,10]$. Such equations have been recently applied to heavy-ion collisions at intermediate energies $[11,12,13]$.

As an alternative to the simulations by kinetic equations, mean-field multi-fluid models have been developed for describing nuclear collisions $[2,3,14,15]$. In these multi-fluid models the local momentum distribution is parametrized by a set of relevant collective quantities. Using local equilibrium distributions for each fluid, one obtains a closed set of equations for the collective variables. By keeping only the description of gross properties of the phase-space distribution, these multi-fluid models have the great advantage of being fast numerically solvable $[14,15]$.

Many-body correlations, other than those leading to the binary collision term, are neglected in all these single-particle models. As is evident, e.g., from the "molecular chaos assumption" in the derivation of such kinetic equations, these models describe an average evolution of an ensemble of systems. Therefore, these models are not appropriate for treating fluctuation phenomena. For the description of fluctuations in the ensemble, an extension of the BUU-type equations is required. Such extensions have been performed in a statistical approximation $[16,17,18$, $19,20,21$, giving rise to generalized fluctuating forces of the Langevin type in the BUU equation. This stochastic BUU equation, referred to as a BoltzmannLangevin equation (BLE), provides a consistent basis for treating dissipation and fluctuation properties of a collision process. For relativistic nuclear collisions this approach was generalized on the basis of the Walecka-type mean-field theory [22] (cf. sect. 2).

Whereas it is with some effort possible to obtain numerical solutions of the BUU equations $[11,12,13]$, it is rather impossible to compute results with sufficiently small statistical errors within the BLE approach. Therefore, we have reduced the relativistic BLE to a stochastic two-fluid model (cf. sect. 3) and a stochastic three-fluid model (cf. appendix). The interflow friction and diffusion coefficients, associated with the collective variables, have been derived. The resulting equations are illustrated by studying effects of fluctuations in the equilibration process for counter-streaming nuclear fluids (cf. sect. 4). The fluctuations are found to be large, the local variances of the relative 4-momentum becoming comparable to its square mean values for intermediate times during equilibration. 


\section{Relativistic Boltzmann-Langevin Equation}

A derivation of the relativistic BLE in the framework of the Walecka-type effective-field theory was presented in ref. [22]. Here we give a brief survey and the main results of the model. Binary collisions play a twofold role during the dynamical evolution of a system: (i) they produce dissipation by randomizing the momentum distribution of the constituent particles and, in addition, (ii) they induce fluctuations by propagating correlations in the phase space. These two effects of the binary collisions, i.e. dissipation and fluctuation, can be incorporated into the equation of motion yielding a stochastic transport equation for the singleparticle density. For the sake of simplicity, we restrict our treatment to elastic binary collisions and consider the semi-classical evolution of a spin-isospin averaged phase-space distribution function *

$$
F(x, p)=\frac{2 d_{N}}{(2 \pi)^{3}} \theta\left(p_{0}\right) \delta\left(p^{2}-M_{N}^{* 2}\right) f(x, \boldsymbol{p}),
$$

where $f(x, \boldsymbol{p})$ (a function of the time $x^{0}$, space $\boldsymbol{x}$ and 3 -momentum $\boldsymbol{p}$ ) is a conventional occupation number, $M_{N}^{*}$ and $d_{N}$ stand for an effective nucleon mass and the nucleon spin-isospin degeneracy factor, respectively. In the semiclassical limit we obtain a stochastic BUU-type equation

$$
\hat{D} \cdot F(x, p)=K(x, p)+\delta K(x, p)
$$

for the fluctuating distribution function $F(x, p)$ with

$$
\hat{D}=p_{\mu} \partial^{\mu}-g_{\omega} p_{\mu} \omega^{\mu \nu} \frac{\partial}{\partial p^{\nu}}+M_{N}^{*}\left(\partial_{\mu} M_{N}^{*}\right) \frac{\partial}{\partial p_{\mu}} .
$$

The left-hand side of eq. (2) describes the Vlasov propagation in terms of the scalar field $\sigma(x)$ and the vector field $\omega_{\mu}(x)$. The $\sigma$ - and $\omega$-fields enter the kinetic equation (2) via the effective nucleon mass $M_{N}^{*}=M_{N}-g_{\sigma} \sigma$ and the field-strength tensor $\omega^{\mu \nu}=\partial^{\mu} \omega^{\nu}-\partial^{\nu} \omega^{\mu}$, cf. $[24,25]$. They are determined by the equations

$$
\begin{gathered}
\left(\square+m_{\sigma}^{2}\right) \sigma+d U / d \sigma=g_{\sigma} \rho, \\
\left(\square+m_{\omega}^{2}\right) \omega_{\mu}=g_{\omega} J_{\mu} .
\end{gathered}
$$

The source terms in eqs. (4) and (5) contain the scalar density $\rho$ and baryon current $J_{\mu}$ which are self-consistently defined in terms of the distribution function,

$$
\begin{aligned}
& \rho(x)=M_{N}^{*} \int d^{4} p F(x, p), \\
& J_{\mu}(x)=\int d^{4} p p_{\mu} F(x, p) .
\end{aligned}
$$

* A generalization to inelastic interactions and to an explicit treatment of isospin degrees of freedom can be naturally performed according to refs. $[2,3,14,15,23]$ 
Equation (4) includes a self-interaction of the $\sigma$-field, which is described by $U(\sigma)=$ $\frac{1}{3} b \sigma^{3}+\frac{1}{4} c \sigma^{4}$.

The collision term, in general, can be written in terms of gain and loss terms,

$$
K(x, p)=\tilde{F}(x, p) G(x, p)-F(x, p) L(x, p)
$$

where

$$
\tilde{F}(x, p)=2 \theta\left(p_{0}\right) \delta\left(p^{2}-M_{N}^{* 2}\right)(1-f(x, p))
$$

is the Pauli factor. An additional term, $\delta K(x, p)$, in eq. (2) presents a fluctuating part of the collision term. Such a contribution always arises in a transport theory whenever one deals with a reduced treatment. It describes a coupling to remaining degrees of freedom, which are not explicitly considered. By analogy with the treatment of the Brownian motion, it is assumed that eq. (2) describes a stochastic process, in which the entire distribution function $F(x, p)$ is a stochastic variable and $\delta K(x, p)$ acts as a random force. In such a description the fluctuating collision term is characterized by a correlation function,

$$
\left\langle\delta K(x, p) \delta K\left(x^{\prime}, p^{\prime}\right)\right\rangle=C\left(x, p ; x^{\prime}, p^{\prime}\right) \delta^{(4)}\left(x-x^{\prime}\right),
$$

which is assumed to be local in space and time.

In the weak-coupling limit together with a quasi-particle approximation, the collision term and the correlation function can be explicitly calculated. The collision term takes a usual BUU form. The gain and loss rates read

$$
\begin{aligned}
& G(1)=\int d^{4} p_{2} d^{4} p_{3} d^{4} p_{4} W(12 \mid 34) \tilde{F}(2) F(3) F(4), \\
& L(1)=\int d^{4} p_{2} d^{4} p_{3} d^{4} p_{4} W(12 \mid 34) F(2) \tilde{F}(3) \tilde{F}(4),
\end{aligned}
$$

where $F(j) \equiv F\left(x, p_{j}\right)$ etc., and $W(12 \mid 34)$ denotes a Lorentz-invariant transition rate. This transition rate is expressed in terms of the scattering cross-section. In the case of elastic scattering, to which we confine ourselves in the present paper, it reads

$$
W(12 \mid 34)=\frac{1}{2} s_{12} \frac{d \sigma}{d \Omega_{c m}} \delta^{(4)}\left(p_{1}+p_{2}-p_{3}-p_{4}\right),
$$

where $s_{12}=\left(p_{1}+p_{2}\right)^{2}$, and $d \sigma / d \Omega_{c m}$ is the differential cross-section in the nucleonnucleon c.m. frame. The correlation function (10) can be expressed as

$$
\begin{aligned}
C\left(1,1^{\prime}\right) & =F(1) L\left(1,1^{\prime}\right) F\left(1^{\prime}\right)+\tilde{F}(1) \tilde{G}\left(1,1^{\prime}\right) \tilde{F}\left(1^{\prime}\right) \\
& -F(1) \tilde{L}\left(1,1^{\prime}\right) \tilde{F}\left(1^{\prime}\right)-\tilde{F}(1) G\left(1,1^{\prime}\right) F\left(1^{\prime}\right) \\
& +\delta^{(4)}\left(p-p^{\prime}\right)[F(1) L(1)+\tilde{F}(1) G(1)] .
\end{aligned}
$$

Here, $F(1)$ and $\tilde{F}(1)$ represent locally averaged distribution functions with $(1) \equiv$ $(x, p)$ and $\left(1^{\prime}\right) \equiv\left(x^{\prime}, p^{\prime}\right)$. The correlation coefficients, $G\left(1,1^{\prime}\right), L\left(1,1^{\prime}\right), \tilde{G}\left(1,1^{\prime}\right)$ 
and $\tilde{L}\left(1,1^{\prime}\right)$, are given by variational derivatives of the gain and loss rates of eqs. (11) and (12) with respect to $F(1)$ and $\tilde{F}(1)$ :

$$
\begin{aligned}
G\left(1,1^{\prime}\right) & =\left[\delta G(1) / \delta F\left(1^{\prime}\right)\right]_{F, \tilde{F}}, \\
\tilde{G}\left(1,1^{\prime}\right) & =\left[\delta G(1) / \delta \tilde{F}\left(1^{\prime}\right)\right]_{F, \tilde{F}}, \\
L\left(1,1^{\prime}\right) & =\left[\delta L(1) / \delta F\left(1^{\prime}\right)\right]_{F, \tilde{F}} \\
\tilde{L}\left(1,1^{\prime}\right) & =\left[\delta L(1) / \delta \tilde{F}\left(1^{\prime}\right)\right]_{F, \tilde{F}},
\end{aligned}
$$

evaluated with the locally averaged distribution functions. The correlation function is closely related to the collision term and is entirely determined by average properties of the single-particle density. The fluctuation and dissipation properties, described by the collision term and the correlation function, are related to each other through a "fluctuation-dissipation theorem". Therefore, the close relationship between the correlation function and the collision term can be regarded as a fluctuation-dissipation theorem associated with the stochastic evolution of the phase-space distribution. The stochastic BUU eq. (2) provides an extended single-particle transport description of the collision process by incorporating the dynamical fluctuations in a statistical approximation. Below, it is referred to as a relativistic BLE.

In order to prepare a basis for a multi-fluid model as in refs. $[2,3,14,15,26$, $27,28,29,30,31,32]$, we introduce a multi-fluid decomposition of the nucleon phase-space distribution function,

$$
F(x, p)=\sum_{\alpha} F^{(\alpha)}(x, p),
$$

where $F^{(\alpha)}(x, p)$ denotes a partial distribution function for an $\alpha$-fluid with $\alpha=p$ and $t$, respectively, for the projectile- and target-like particles in the two-fluid dynamics $[14,26,27,28,29,30]$, and in addition $\alpha=s$ for "stopped" particles in the three-fluid model $[15,31,32]$. It is worthwhile to note that the relativistic BLE in the form of eq. (2) describes a system of identical particles. The decomposition (16) means that at each spatial point we subdivide the identical nucleons into different groups according their position in the momentum space. This approach is similar to a kinetic treatment of a mixture of different (nonidentical) particles, when each species is characterized by its own distribution function. An essential difference of the present case consists in the fact that we cannot decompose the Pauli factors $\tilde{F}(x, p)$ in the same way as that in eq. (16), since the Pauli blocking is determined by all the $\alpha$-components due to the identity of particles. Nevertheless, for the sake of convenience we shall use notation $\tilde{F}^{(\alpha)}(x, p)$ for the Pauli factors, keeping in mind that $\tilde{F}^{(\alpha)} \equiv \tilde{F}$. The following set of coupled BLE's for the fluctuating partial distribution functions $F^{(\alpha)}(x, p)$ can be obtained:

$$
\hat{D} \cdot F^{(\alpha)}(x, p)=K^{(\alpha)}(x, p)+\delta K^{(\alpha)}(x, p),
$$

where the $\hat{D}$ operator is defined by eq. (3) and the collision term is given by

$$
K^{(\alpha)}(x, p)=-F^{(\alpha)}(x, p) L^{(\alpha)}(x, p)+\tilde{F}^{(\alpha)}(x, p) G^{(\alpha)}(x, p)
$$


with the gain and loss rates

$$
\begin{aligned}
& G^{(\alpha)}(1)=\sum_{\beta \gamma \delta} \int d^{4} p_{2} d^{4} p_{3} d^{4} p_{4} W(1 \alpha 2 \beta \mid 3 \gamma 4 \delta) \tilde{F}^{(\beta)}(2) F^{(\gamma)}(3) F^{(\delta)}(4) \\
& L^{(\alpha)}(1)=\sum_{\beta \gamma \delta} \int d^{4} p_{2} d^{4} p_{3} d^{4} p_{4} W(1 \alpha 2 \beta \mid 3 \gamma 4 \delta) F^{(\beta)}(2) \tilde{F}^{(\gamma)}(3) \tilde{F}^{(\delta)}(4)
\end{aligned}
$$

Here $W(1 \alpha 2 \beta \mid 3 \gamma 4 \delta)$ denotes the transition rate between a pair of single-particle states with momenta $p_{1}$ and $p_{2}$ from the $\alpha$ - and $\beta$-components of the system, respectively, and another pair of states from the $\gamma$ - and $\delta$-components with momenta $p_{3}$ and $p_{4}$. The decomposition of the total transition rate into partial rates,

$$
W(1 \alpha 2 \beta \mid 34)=\sum_{\gamma \delta} W(1 \alpha 2 \beta \mid 3 \gamma 4 \delta),
$$

is necessary in the further formulation of multi-fluid models (cf. sect. 3 for the two-fluid model and the appendix for the three-fluid model).

The fluctuating part of the collision term in eq. (17) is specified by a correlation matrix

$$
\left\langle\delta K^{(\alpha)}(x, p) \delta K^{(\beta)}\left(x^{\prime}, p^{\prime}\right)\right\rangle=C^{(\alpha \beta)}\left(x, p ; x^{\prime}, p^{\prime}\right) \delta^{(4)}\left(x-x^{\prime}\right) .
$$

Its elements are defined as follows

$$
\begin{aligned}
C^{(\alpha \beta)}\left(1,1^{\prime}\right) & =F^{(\alpha)}(1) L^{(\alpha \beta)}\left(1,1^{\prime}\right) F^{(\beta)}\left(1^{\prime}\right)+\tilde{F}^{(\alpha)}(1) \tilde{G}^{(\alpha \beta)}\left(1,1^{\prime}\right) \tilde{F}^{(\beta)}\left(1^{\prime}\right) \\
& -F^{(\alpha)}(1) \tilde{L}^{(\alpha \beta)}\left(1,1^{\prime}\right) \tilde{F}^{(\beta)}\left(1^{\prime}\right)-\tilde{F}^{(\alpha)}(1) G^{(\alpha \beta)}\left(1,1^{\prime}\right) F^{(\beta)}\left(1^{\prime}\right) \\
& +\delta\left(p-p^{\prime}\right) \delta_{\alpha \beta}\left[F^{(\alpha)}(1) L^{(\alpha)}(1)+\tilde{F}^{(\alpha)}(1) G^{(\alpha)}(1)\right] .
\end{aligned}
$$

Similar to eqs. (15), the correlation coefficients are defined in terms of the gain and loss rates

$$
\begin{aligned}
& G^{(\alpha \beta)}\left(1,1^{\prime}\right)=\left[\delta G^{(\alpha)}(1) / \delta F^{(\beta)}\left(1^{\prime}\right)\right]_{F, \tilde{F}}, \\
& \tilde{G}^{(\alpha \beta)}\left(1,1^{\prime}\right)=\left[\delta G^{(\alpha)}(1) / \delta \tilde{F}^{(\beta)}\left(1^{\prime}\right)\right]_{F, \tilde{F}}, \\
& L^{(\alpha \beta)}\left(1,1^{\prime}\right)=\left[\delta L^{(\alpha)}(1) / \delta F^{(\beta)}\left(1^{\prime}\right)\right]_{F, \tilde{F}}, \\
& \tilde{L}^{(\alpha \beta)}\left(1,1^{\prime}\right)=\left[\delta L^{(\alpha)}(1) / \delta \tilde{F}^{(\beta)}\left(1^{\prime}\right)\right]_{F, \tilde{F}},
\end{aligned}
$$

evaluated with the locally averaged partial distribution functions.

\section{Stochastic Two-Fluid Model}

So far the set of kinetic equations (17) is equivalent to the original equation (2) and the solution of the set of eqs. (17) is not easier than that of the original eq. (2). To simplify the problem, we introduce a Fermi-Dirac ansatz for the partial distribution functions,

$$
F^{(\alpha)}(x, p)=\frac{2 d_{N}}{(2 \pi)^{3}} \delta\left(p^{2}-M_{N}^{* 2}\right) \theta\left(p^{0}\right)\left[\exp \left\{\left(u^{(\alpha)} \cdot p-\nu^{(\alpha)}\right) / T^{(\alpha)}\right\}+1\right]^{-1}
$$


where $\alpha=p, t$ (as well as $s$ in the case of a three-fluid model). The quantities $u^{(\alpha)}(x), T^{(\alpha)}(x)$ and $\nu^{(\alpha)}(x)$ denote the local 4-velocity, temperature and effective nucleon chemical potential of the $\alpha$-fluid, respectively, and have to be determined from the kinetic equations. The 4-velocities are normalized by the condition $u_{\mu}^{(\alpha)} u^{(\alpha) \mu}=1$.

In this section we perform a reduction of the relativistic BLE to a stochastic two-fluid model. The results of the same reduction to a stochastic three-fluid model are presented in the Appendix.

Taking the first two moments of eqs. (17)

$$
\begin{gathered}
\int d^{4} p \hat{D} \cdot F^{(\alpha)}, \\
\int d^{4} p p_{\nu} \hat{D} \cdot F^{(\alpha)}
\end{gathered}
$$

with respect to the 4-momentum, we have the required number of linearly independent equations for the parameters of the model distribution functions (25). With the explicit form of the $\hat{D}$ operator, we obtain these equations in the form

$$
\begin{gathered}
\partial^{\mu} J_{\mu}^{(\alpha)}=0 \\
\partial^{\mu} T_{\mu \nu}^{(\alpha)}-g_{\omega} J_{\mu}^{(\alpha)} \omega^{\mu}{ }_{\nu}+g_{\sigma} \rho^{(\alpha)} \partial_{\nu} \sigma=R_{\nu}^{(\alpha)}+\delta R_{\nu}^{(\alpha)}
\end{gathered}
$$

where $\rho^{(\alpha)}, J_{\mu}^{(\alpha)}$ and $T_{\mu \nu}^{(\alpha)}$ are, respectively, the scalar density, baryonic current and energy-momentum tensor of the $\alpha$-fluid:

$$
\begin{gathered}
\rho^{(\alpha)}(x)=M_{N}^{*} \int d^{4} p F^{(\alpha)}(x, p), \\
J_{\mu}^{(\alpha)}(x)=\int d^{4} p p_{\mu} F^{(\alpha)}(x, p)=n^{(\alpha)}(x) u_{\mu}^{(\alpha)}(x), \\
T_{\mu \nu}^{(\alpha)}(x)=\int d^{4} p p_{\mu} p_{\nu} F^{(\alpha)}(x, p) \\
=\left(E^{(\alpha)}+P^{(\alpha)}\right) u_{\mu}^{(\alpha)} u_{\nu}^{(\alpha)}-P^{(\alpha)} g_{\mu \nu} .
\end{gathered}
$$

These quantities are defined via the model distribution functions (25) in terms of their parameters. The pressure $P^{(\alpha)}$, the internal energy density $E^{(\alpha)}$, the partial scalar density $\rho^{(\alpha)}$ and the local-rest-frame density $n^{(\alpha)}$ of the $\alpha$-fluid are expressed by standard statistical relations in terms of the corresponding temperature, effective chemical potentials and effective baryon masses, cf. [33]. The terms of collisional interfluid interaction are expressed as

$$
\begin{gathered}
R_{\nu}^{(\alpha)}=\int d^{4} p p_{\nu} K^{(\alpha)} \\
\left\langle\delta R_{\mu}^{(\alpha)}(x) \delta R_{\nu}^{(\beta)}\left(x^{\prime}\right)\right\rangle=\delta^{(4)}\left(x-x^{\prime}\right) \int d^{4} p \int d^{4} p^{\prime} p_{\mu} p_{\nu}^{\prime} C^{(\alpha \beta)}\left(x, p ; x^{\prime}, p^{\prime}\right) .
\end{gathered}
$$

Equations (28) and (29) have a conventional hydrodynamical meaning. They describe the local balance of the number of particles and the 4-momentum in the 
interacting fluids. To make this set of equations a closed one, one should express the interfluid interaction, $R_{\nu}^{(\alpha)}$ and $\delta R_{\nu}^{(\alpha)}$, in terms of the parameters of the partial distribution functions (25). By using the explicit forms of the collision term $K^{(\alpha)}$ and the correlation matrix $C^{(\alpha \beta)}$, cf. eqs. (18)-(24), one obtains the following expressions for the average friction force, $R_{\nu}^{(\alpha)}$, and the corresponding correlation matrix of the stochastic force (34):

$$
\begin{aligned}
& R_{\nu}^{(\alpha)}(x)=\int d^{4} p_{1} d^{4} p_{2} d^{4} p_{3} d^{4} p_{4} W(1 \alpha 2 \bar{\alpha} \mid 3 \alpha 4 \bar{\alpha}) \\
& \times\left(p_{3}-p_{1}\right)_{\nu} F^{(\alpha)}(1) F^{(\bar{\alpha})}(2) \tilde{F}(3) \tilde{F}(4) \\
&\left\langle\delta R_{\mu}^{(\alpha)}(x) \delta R_{\nu}^{(\alpha)}\left(x^{\prime}\right)\right\rangle=\delta^{(4)}\left(x-x^{\prime}\right) \int d^{4} p_{1} d^{4} p_{2} d^{4} p_{3} d^{4} p_{4} W(1 \alpha 2 \bar{\alpha} \mid 3 \alpha 4 \bar{\alpha}) \\
& \times\left(p_{3}-p_{1}\right)_{\mu}\left(p_{3}-p_{1}\right)_{\nu} F^{(\alpha)}(1) F^{(\bar{\alpha})}(2) \tilde{F}(3) \tilde{F}(4), \\
&\left\langle\delta R_{\mu}^{(\alpha)}(x) \delta R_{\nu}^{(\bar{\alpha})}\left(x^{\prime}\right)\right\rangle=-\left\langle\delta R_{\mu}^{(\alpha)}(x) \delta R_{\nu}^{(\alpha)}\left(x^{\prime}\right)\right\rangle
\end{aligned}
$$

where $\bar{\alpha}=t$ if $\alpha=p$, while $\bar{\alpha}=p$ if $\alpha=t$.

In principle, eqs. (35)-(37) solve the problem of relating the interfluid coupling to the characteristics of the fluids. One can calculate the multidimensional integrals of eqs. (35) and (36) at each step of the two-fluid simulation and, thus, determine the interfluid coupling. To simplify this task, a further approximation is introduced according to $[2,3,14,26,27,28,29,30]$. Proceeding from the conservation laws for the nucleon-nucleon scattering and from symmetry considerations, the friction force (35) and the correlator of its stochastic part (36) is expressed as

$$
\begin{aligned}
R_{\nu}^{(\alpha)}(x)= & -M_{N}^{*} \int d^{4} p_{1} d^{4} p_{2} D\left(p_{1}-p_{2}\right)_{\nu} F^{(\alpha)}(1) F^{(\bar{\alpha})}(2), \\
\left\langle\delta R_{\mu}^{(\alpha)}(x) \delta R_{\nu}^{(\alpha)}\left(x^{\prime}\right)\right\rangle= & \frac{1}{2} \delta^{(4)}\left(x-x^{\prime}\right) M_{N}^{*} \sum_{\beta} \int d^{4} p_{1} d^{4} p_{2} F^{(\beta)}(1) F^{(\bar{\beta})}(2) \\
& \times\left\{\left(A_{\|}-A_{\perp}\right)\left(p_{1}-p_{2}\right)_{\mu}\left(p_{1}-p_{2}\right)_{\nu}+A_{\perp} g_{\mu \nu}\left(p_{1}-p_{2}\right)^{2}\right\}
\end{aligned}
$$

where

$$
\left(\begin{array}{c}
D \\
A_{\|} \\
A_{\perp}
\end{array}\right)=\frac{1}{M_{N}^{*}} \int d^{4} p_{3} d^{4} p_{4} W(1 p 2 t \mid 3 p 4 t) \tilde{F}(3) \tilde{F}(4)\left(\begin{array}{c}
1-\cos \theta_{c m} \\
\frac{1}{2}\left(1-\cos \theta_{c m}\right)^{2} \\
\frac{1}{6} \sin ^{2} \theta_{c m}
\end{array}\right)
$$

and $\theta_{c m}$ is the scattering angle of two nucleons in their center-of-mass frame. By means of the explicit form of the transition rate, cf. eq. (13), we transform these transport coefficiens to a more transparent form

$$
\left(\begin{array}{c}
D \\
A_{\|} \\
A_{\perp}
\end{array}\right)=\frac{1}{4 M_{N}^{*}}\left[s_{12}\left(s_{12}-4 M_{N}^{* 2}\right)\right]^{1 / 2}\left(\begin{array}{c}
\sigma_{t r}(\bar{s}) \\
\frac{1}{2} \sigma_{\|}(\bar{s}) \\
\frac{1}{6} \sigma_{\perp}(\bar{s})
\end{array}\right)
$$


where the corresponding cross sections are defined as follows

$$
\left(\begin{array}{c}
\sigma_{t r} \\
\sigma_{\|} \\
\sigma_{\perp}
\end{array}\right)=\int_{\theta_{c m}<\pi / 2} d \sigma(1-f(3))(1-f(4))\left(\begin{array}{c}
1-\cos \theta_{c m} \\
\left(1-\cos \theta_{c m}\right)^{2} \\
\sin ^{2} \theta_{c m}
\end{array}\right) .
$$

The transport coefficients $D, A_{\|}$and $A_{\perp}$ can be approximately treated as functions of one variable $s_{12}=\left(p_{1}+p_{2}\right)^{2}$, only, thereby neglecting the weak dependence on the individual momenta $p_{1}$ and $p_{2}$ from the Pauli factors $(1-f(3))(1-f(4))$ in the integrals of eq. (42). Assuming the cross-section to be a sufficiently smooth function of the incident energy, we find that the transport coefficients $D, A_{\|}$and $A_{\perp}$ appear to be slowly varying functions of $s_{12}$ as compared to the $F(1) F(2)$ product. Due to this slow variation we replace $D, A_{\|}$and $A_{\perp}$ in the integrals by constants obtained for some intermediate value,

$$
\bar{s}=M_{N}^{* 2}\left\{\left(u^{(p)}+u^{(t)}\right)^{2}+2\left(u^{(p)} \cdot u^{(t)}\right)\left[\left(n^{(p)} / \rho^{(p)}\right)\left(n^{(t)} / \rho^{(t)}\right)-1\right]\right\},
$$

of the $s_{12}$ argument. Such a choice for $\bar{s}$ was analyzed in refs. $[14,15]$ and found to be reasonably good. With this approximation the integrals can be immediately calculated. The result reads

$$
\begin{gathered}
R_{\nu}^{(\alpha)}(x)=-\bar{D}\left(J_{\nu}^{(\alpha)} \rho^{(\bar{\alpha})}-\rho^{(\alpha)} J_{\nu}^{(\bar{\alpha})}\right) \\
\left\langle\delta R_{\mu}^{(\alpha)}(x) \delta R_{\nu}^{(\alpha)}\left(x^{\prime}\right)\right\rangle= \\
=\delta^{(4)}\left(x-x^{\prime}\right) \sum_{\beta}\left\{\left(\bar{A}_{\|}-\bar{A}_{\perp}\right)\left(T_{\mu \nu}^{(\beta)} \rho^{(\bar{\beta})}-M_{N}^{*} J_{\mu}^{(\beta)} J_{\nu}^{(\bar{\beta})}\right)\right. \\
\left.+\bar{A}_{\perp} g_{\mu \nu} M_{N}^{*}\left(\rho^{(\beta)} \rho^{(\bar{\beta})}-J^{(\beta)} \cdot J^{(\bar{\beta})}\right)\right\}
\end{gathered}
$$

with $\bar{D}=D(\bar{s})$, etc.

Now we can summarize the derived equations of the stochastic two-fluid model. The average interfluid friction force (44) and the correlation matrix of the stochastic force, as given by eqs. (37) and (45), are completely determined by the transport coefficients and the $\rho^{(\alpha)}, J_{\mu}^{(\alpha)}$ and $T_{\mu \nu}^{(\alpha)}$ variables of the two-fluid model. Thus, we have obtained a closed set of stochastic equations consisting of eqs. (4), (5), (28), (29), (37), (44) and (45). The transport coefficients are determined by eq. (40) in terms of cross sections and Pauli-blocking factors at the value of the $s_{12}$ argument specified by eq. (43). Like the BLE the stochastic two-fluid model only needs the mean-field parameters and the cross sections as input.

The derived model presents a multidimensional stochastic problem, since here we have more than one random force. To treat such a problem, one should, first, diagonalize it in the space of random forces [34], i.e. transform the variables in such a way that the correlation matrix [see eqs. (37) and (45)] becomes diagonal. The diagonalization in the space of "fluids" can be done straightforwardly. Let us define new stochastic variables as follows

$$
\begin{aligned}
& \rho^{( \pm)}=\rho^{(p)} \pm \rho^{(t)}, \\
& J_{\mu}^{( \pm)}=J_{\mu}^{(p)} \pm J_{\mu}^{(t)},
\end{aligned}
$$




$$
T_{\mu \nu}^{( \pm)}=T_{\mu \nu}^{(p)} \pm T_{\mu \nu}^{(t)}
$$

Equations in terms of these new variables can be obtained by taking sums and differences of eqs. (28) and (29) for $\alpha=p$ and $\alpha=t$ :

$$
\begin{gathered}
\partial^{\mu} J_{\mu}^{( \pm)}=0 \\
\partial^{\mu} T_{\mu \nu}^{( \pm)}-g_{\omega} J_{\mu}^{( \pm)} \omega^{\mu}{ }_{\nu}+g_{\sigma} \rho^{( \pm)} \partial_{\nu} \sigma=R_{\nu}^{( \pm)}+\delta R_{\nu}^{( \pm)}
\end{gathered}
$$

In this ( \pm )-representation we have

$$
\begin{gathered}
R_{\nu}^{(+)}=\delta R_{\nu}^{(+)}=0 \\
R_{\nu}^{(-)}=2 R_{\nu}^{(p)} \\
\left\langle\delta R_{\mu}^{(-)}(x) \delta R_{\nu}^{(-)}\left(x^{\prime}\right)\right\rangle=4\left\langle\delta R_{\mu}^{(p)}(x) \delta R_{\nu}^{(p)}\left(x^{\prime}\right)\right\rangle .
\end{gathered}
$$

The (+)-subset of equations takes care of the global conservation laws in the system. Hence, it does not include any terms of the collisional interfluid coupling. The (-)-subset describes the dissipative interaction of two fluids and, hence, contains random forces. The correlation tensor (53) is diagonalized in the 4-dimensional space. Note that in the particular case of two interacting fluids of the same density and temperature, this tensor is already diagonal in the equal-velocity frame $\left(\boldsymbol{u}^{(p)}=-\boldsymbol{u}^{(t)}\right)$.

\section{Equilibration of Counter-Streaming Fluids}

To illustrate the stochastic two-fluid model of sect. 3 and to study qualitative effects of fluctuations, we consider the equilibration in a homogeneous nuclear system. It is assumed that initially there are two cold $\left(T^{(p)}=0\right.$ and $\left.T^{(t)}=0\right)$ spatially homogeneous counter-streaming nuclear fluids of normal nuclear density $\left(n_{0}\right)$. We follow the time evolution of the system starting from the moment the collisional coupling between the fluids is turned on. Such a simplified problem has already been treated within multi-fluid models $[14,15]$ in order to compare the multi-fluid description with the complete solution of the kinetic equation $[6,7]$. For the sake of simplicity, we consider here the case of zero mean fields $\left(g_{\sigma}=g_{\omega}=0\right)$.

In general, auto-correlation functions of the fluid variables can be directly calculated by a numerical simulation of the stochastic multi-fluid equations. From these auto-correlation functions one can deduce a correlation volume, i.e. the volume in which the fluctuations of the fluid variables are correlated. Here we do not solve the multi-fluid equations to a full extent, but rather want to estimate a magnitude of the local fluctuations. For this purpose, we perform a coarse graining of the system in cells of the correlation volume in the equal-velocity frame. This volume is estimated proceeding from the fact that the correlation length is of the order of the mean free path. In our case the mean free path is determined by the drift coefficient $\bar{D}$ or, equivantly, by the transport cross section $\sigma_{t r}$, cf. eqs. (41) and (42),

$$
l=1 /\left(n \sigma_{t r}\right),
$$


where $n=J_{0}^{(\alpha)}$ is the density of one of the fluids. (Note, the densities of both fluids are equal in the equal-velocity frame in our case.) Here we take only the density of one fluid, since only collisions between particles of different fluids are relevant to the two-fluid evolution. The longitudinal size of the correlation volume is estimated as

$$
l_{\|}=l\left(\cos \theta_{c m}\right\rangle
$$

where $\left\langle\cos \theta_{c m}\right\rangle=\left(\sigma_{t o t}-\sigma_{t r}\right) / \sigma_{t o t}$, cf. eq. (42), and $\sigma_{t o t}$ is the total Pauli-blocked cross section. Correspondingly, the transverse area of the correlation volume is

$$
S_{\perp}=\pi l^{2}\left\langle\sin ^{2} \theta_{c m}\right\rangle
$$

with $\left\langle\sin ^{2} \theta_{c m}\right\rangle=\sigma_{\perp} / \sigma_{\text {tot }}$, cf. eq. (42). Thus, the correlation volume is estimated as a cylinder with area $S_{\perp}$ and length $l_{\|}$, yielding the volume

$$
V_{\text {corr }} \approx S_{\perp} l_{\|}=\pi l^{3} \frac{\sigma_{\text {tot }}-\sigma_{\text {tr }}}{\sigma_{\text {tot }}} \frac{\sigma_{\perp}}{\sigma_{\text {tot }}} \text {. }
$$

Because of the original anisotropy of the system in the momentum space, the correlation volume is streched along the "beam" direction.

Having such a coarse graining, we can employ the technique of refs. [35]. We integrate equations of motion (28) and (29) over the cell volume in the equalvelocity reference frame. Thus, we have the following integral variables of the cell: the number of nucleons in the cell,

$$
N^{(\alpha)}=\int_{V_{\text {corr }}} d^{3} x J_{0}^{(\alpha)}
$$

and the energy-momentum flux,

$$
P_{\nu}^{(\alpha)}=\frac{1}{N^{(\alpha)}} \int_{V_{\text {corr }}} d^{3} x T_{0 \nu}^{(\alpha)} \equiv \epsilon^{(\alpha)} u_{\nu}^{(\alpha)}
$$

where $\epsilon^{(\alpha)}$ is an average internal energy per nucleon in the $\alpha$-fluid and $u_{\nu}^{(\alpha)}$ stands for an average $\alpha$-fluid 4 -velocity. The corresponding equations of motion are

$$
\begin{gathered}
\frac{d}{d t} N^{(\alpha)}=0 \\
N^{(\alpha)} \frac{d}{d t} P_{\nu}^{(\alpha)}=-\overline{\mathcal{D}}\left(P_{\nu}^{(\alpha)}-P_{\nu}^{(\bar{\alpha})}\right)+\delta \bar{R}_{\nu}^{(\alpha)} .
\end{gathered}
$$

The correlator of the random force in the cell, $\delta \bar{R}_{\nu}^{(\alpha)}$, is obtained straightforwardly as

$$
\begin{aligned}
& \left\langle\delta \bar{R}_{\mu}^{(\alpha)}(t) \delta \bar{R}_{\nu}^{(\alpha)}\left(t^{\prime}\right)\right\rangle=\int_{V_{\text {corr }}} d^{3} x\left\langle\delta R_{\mu}^{(\alpha)}(x) \delta R_{\nu}^{(\alpha)}\left(x^{\prime}\right)\right\rangle \\
& \quad=\delta\left(t-t^{\prime}\right)\left\{\left(\overline{\mathcal{A}}_{\|}-\overline{\mathcal{A}}_{\perp}\right)\left(P^{(p)}-P^{(t)}\right)_{\mu}\left(P^{(p)}-P^{(t)}\right)_{\nu}+2 \overline{\mathcal{A}}_{\perp} g_{\mu \nu}\left(M_{N}^{2}-P^{(p)} \cdot P^{(t)}\right)\right\}
\end{aligned}
$$

$$
\left\langle\delta \bar{R}_{\mu}^{(\alpha)}(t) \delta \bar{R}_{\nu}^{(\bar{\alpha})}\left(t^{\prime}\right)\right\rangle=-\left\langle\delta \bar{R}_{\mu}^{(\alpha)}(t) \delta \bar{R}_{\nu}^{(\alpha)}\left(t^{\prime}\right)\right\rangle
$$


The appropriate transport coefficients are

$$
\left(\begin{array}{c}
\overline{\mathcal{D}} \\
\overline{\mathcal{A}}_{\| 1} \\
\overline{\mathcal{A}}_{\perp}
\end{array}\right)=\frac{M_{N}}{P_{0}^{(p)} P_{0}^{(t)}} \int_{V_{c o r r}} d^{3} x J_{0}^{(p)}(x) J_{0}^{(t)}(x)\left(\begin{array}{c}
\bar{D} \\
\bar{A}_{\| 1} \\
\bar{A}_{\perp}
\end{array}\right) .
$$

In deriving eqs. (61) and (62) the approximate relation, $\rho^{(\alpha)} \approx M_{N} n^{(\alpha)} / \epsilon^{(\alpha)}$, was used, which is valid at not too high excitation energies $\left(\epsilon^{(\alpha)}-M_{N} \ll M_{N}\right)$.

When performing the averaging over a cell, we have assumed that fluctuations in different cells are statistically independent. As seen from eq. (60), the numbers of particles in the cell, $N^{(\alpha)}$, do not fluctuate. They could fluctuate only due to an exchange between neighbouring cells but they do not, since the fluctuations in the neighboring cells would, otherwise, be correlated. Hence, we have $N^{(p)}=N^{(t)}=$ $N$, since we consider interpenetration of two fluids of equal density. One can see that due to relation (63) eq. (61) gives the exact conservation law

$$
\frac{d}{d t}\left(P_{\nu}^{(p)}+P_{\nu}^{(t)}\right)=0
$$

Below, we solve eqs. (60) - (64) up to linear order in the fluctuations. In addition, we neglect the terms of the order of $\left(\epsilon^{(\alpha)}-M_{N}\right) / M_{N}$ as compared to 1 , if this does not violate the conservation law.

First, let us consider the quantity $P_{0}^{(p)} P_{0}^{(t)}$, since it is included in the definition of the new transport coefficients (64). ¿From eqs. (61) one obtains

$$
N \frac{d}{d t}\left(P_{0}^{(p)} P_{0}^{(t)}\right)=-\overline{\mathcal{D}}\left(P_{0}^{(p)}-P_{0}^{(t)}\right)^{2}+\left(P_{0}^{(p)} \delta \bar{R}_{0}^{(t)}+P_{0}^{(t)} \delta \bar{R}_{0}^{(p)}\right) .
$$

The correlator of the random force in eq. (66) is

$$
\left\langle\left(P_{0}^{(p)} \delta \bar{R}_{0}^{(t)}+P_{0}^{(t)} \delta \bar{R}_{0}^{(p)}\right)^{2}\right\rangle=\left(P_{0}^{(p)}-P_{0}^{(t)}\right)^{2}\left\langle\left(\delta \bar{R}_{0}^{(p)}\right)^{2}\right\rangle .
$$

Due to the fact that we have two interpenetrating fluids, which are identical, it follows that $\left\langle\left(P_{0}^{(p)}-P_{0}^{(t)}\right)\right\rangle=0$. Therefore, the r.h.s. of eq. $(66)$ is of second order in the fluctuations, and hence in linear approximation, the $\left(P_{0}^{(p)} P_{0}^{(t)}\right)$ product is a conserved and nonfluctuating quantity,

$$
P_{0}^{(p)} P_{0}^{(t)}=\left(P_{0}^{(p)} P_{0}^{(t)}\right)_{t=0}=E_{c m}^{2},
$$

where $E_{c m}$ is the initial energy per nucleon in the equal-velocity (center-of-mass) frame. Now, using the definitions of transport coefficients of eqs. (41) and (64), we obtain the following expressions

$$
\left(\begin{array}{c}
\overline{\mathcal{D}} \\
\overline{\mathcal{A}}_{\|} \\
\overline{\mathcal{A}}_{\perp}
\end{array}\right)=\frac{N n_{0}}{4 E_{c m} M_{N}}\left[\bar{s}\left(\bar{s}-4 M_{N}^{2}\right)\right]^{1 / 2}\left(\begin{array}{c}
\sigma_{t r}(\bar{s}) \\
\frac{1}{2} \sigma_{\|}(\bar{s}) \\
\frac{1}{6} \sigma_{\perp}(\bar{s})
\end{array}\right)
$$

with

$$
\bar{s}=\left(P^{(p)}+P^{(t)}\right)^{2}+\left(2 M_{N}^{2}-\epsilon^{(p) 2}-\epsilon^{(t) 2}\right)
$$


for the $\bar{s}$-argument of the transport coefficients, defined by eq. (43).

Below we assume that $\overline{\mathcal{D}}$ is constant as a function of the $\bar{s}$ argument. The quality of this approximation is illustrated in fig. 1 . The transport coefficients $\overline{\mathcal{D}}$ and $\overline{\mathcal{A}}_{\|}$are calculated from eq. (69). We use eq. (42) for the calculation of the cross sections of interest. In doing so, we use the Cugnon parametrization [36] of the elastic and inelastic nucleon-nucleon cross sections and replace the factor $\cos \theta_{c m}$ by $\boldsymbol{p}_{m} \cdot \boldsymbol{p}_{c m}^{\prime} / \boldsymbol{p}_{c m}^{2}$, where $\boldsymbol{p}_{c m}$ and $\boldsymbol{p}_{c m}^{\prime}$ are 3-momenta of the incident and scattered particles in the center-of-mass frame, respectively. For inelastic scattering such a modification of the cross sections is a natural extension [14, 15, 28, 29]. The transport coefficients $\overline{\mathcal{D}}$ and $\overline{\mathcal{A}}_{\|}$calculated with and without Pauli blocking, are presented in fig. 1 as functions of an effective kinetic energy $E_{l a b}=\left(\bar{s}-4 M_{N}^{2}\right) / 2 M_{N}$ in the laboratory frame. Whereas $\bar{s}_{\text {in }}$ is an initial value corresponding to the true initial energy $E_{l a b}=1 \mathrm{GeV} / \mathrm{u}, \bar{s}_{f i n}$ is a final value of the $\bar{s}$ argument, corresponding to complete stopping. It is calculated by means of the conservation law (65) and the expression (70). The calculation, taking into account the Pauli blocking, assumes that there are two cold Fermi distributions. This is an upper estimate of the Pauli-blocking effect, since at finite temperatures it becomes less important. As one can see, the $\overline{\mathcal{D}}$ and $\overline{\mathcal{A}}_{\|}$transport coefficients are only slightly affected by the Pauli blocking for $E_{l a b}>500 \mathrm{MeV} / \mathrm{u}$. We also see that the assumption of the constancy of $\overline{\mathcal{D}}$ is a rather poor approximation. However, we accept it, since it gives us a possibility of a simple analytical treatment of our equations.

Having done this preliminary work, we can consider an equation for $P_{\nu}=$ $P_{\nu}^{(p)}-P_{\nu}^{(t)}$. Taking the difference of eq. (61) for $\alpha=p$ and $\alpha=t$, we find

$$
\frac{d}{d t} P_{\nu}=-\frac{1}{2 \tau} P_{\nu}+\frac{1}{N} \delta \bar{R}_{\nu}
$$

with $\tau=(4 \overline{\mathcal{D}} / N)^{-1}$ and

$$
\left\langle\delta \bar{R}_{\mu}(t) \delta \bar{R}_{\nu}\left(t^{\prime}\right)\right\rangle=4 \delta\left(t-t^{\prime}\right)\left\{\left(\overline{\mathcal{A}}_{\|}-\overline{\mathcal{A}}_{\perp}\right) P_{\mu} P_{\nu}+\overline{\mathcal{A}}_{\perp} g_{\mu \nu} P^{2}\right\}
$$

for the correlator of the random force. The transport coefficients $\overline{\mathcal{A}}_{\|}, \overline{\mathcal{A}}_{\perp}$ and $\tau$ in this equation are considered to be constant in time. In deriving eq. (72) we again have neglected all terms of order $\left(\epsilon^{(\alpha)}-M_{N}\right) / M_{N}$.

Multiplying eq. (71) by $P^{\nu}$, we obtain the equation

$$
\frac{d}{d t} P^{2}=-\frac{1}{\tau} P^{2}+\frac{2}{N} P \cdot \delta \bar{R}
$$

for $P^{2}$. Here, the quantity $P \cdot \delta \bar{R}$ is not exactly a "random force", because its mean value does not vanish. Using the formal solution of eq. (71)

$$
P_{\nu}(t)=-\left(P_{\nu}\right)_{t=0} \exp (-t / 2 \tau)+\frac{1}{N} \int_{0}^{t} \exp \left\{\left(t^{\prime}-t\right) / 2 \tau\right\} \delta \bar{R}_{\nu}\left(t^{\prime}\right) d t^{\prime}
$$

we find

$$
\langle P \cdot \delta \bar{R}\rangle=\frac{2}{N}\left(\overline{\mathcal{A}}_{\|}+3 \overline{\mathcal{A}}_{\perp}\right)\left\langle P^{2}\right\rangle=\frac{2}{N} \overline{\mathcal{D}}\left\langle P^{2}\right\rangle=\frac{\left\langle P^{2}\right\rangle}{2 \tau} .
$$


Equation (73) is now written in the integral form

$$
P^{2}(t)=-4 p_{c m}^{2} \exp (-t / \tau)+\frac{2}{N} \int_{0}^{t} \exp \left\{\left(t^{\prime}-t\right) / \tau\right\} P\left(t^{\prime}\right) \cdot \delta \bar{R}\left(t^{\prime}\right) d t^{\prime}
$$

where $p_{c m}\left[=\left(E_{c m}^{2}-M_{N}^{2}\right)^{1 / 2}\right]$ is the initial momentum per nucleon in the equalvelocity reference frame coinciding with the center-of-mass one in our case. The mean value and dispersion of $P^{2}$ is readily obtained as

$$
\begin{gathered}
\left\langle P^{2}\right\rangle=-4 p_{c m}^{2} \exp (-t / \tau)\left(1+\frac{t}{N \tau}\right), \\
\left\langle\left(P^{2}-\left\langle P^{2}\right\rangle\right)^{2}\right\rangle^{1 / 2}=\frac{16 p_{c m}^{2} \overline{\mathcal{A}}_{\|}^{1 / 2}}{N} t^{1 / 2} \exp (-t / \tau),
\end{gathered}
$$

in linear order of the fluctuations.

An interesting feature of the solution for $P^{2}(t)$ consists in a modification of its mean evolution due to fluctuations. The solution for $P^{2}(t)$ without any fluctuations is given by eq. (77) without the $(1+t / N \tau)$ factor. Physically, this factor results from squaring the fluctuating $P_{\nu}(t)$ values. As one can see from the solution (74) for $P_{\nu}(t)$, their mean values are not shifted with respect to the nonfluctuating ones. The $\left\langle P^{2}(t)\right\rangle$ value contains not only the mean evolution of the $P_{\nu}(t)$ components but also their dispersion. Thus $P^{2}(t)$ is an example for a quantity, whose mean value is affected by fluctuations. Indeed, the average values of particle production (e.g. strange particles) and two-particle correlations, which are quadratic in distribution functions, can be strongly affected by fluctuations [37].

Equations (77) and (78) are illustrated in fig. 2. The initial values for $E_{c m}$ and $p_{c m}$ correspond to a nucleus-nucleus collision at $E_{l a b}=1 \mathrm{GeV} / \mathrm{u}$. For the calculation we have taken the transport coefficients (cf. fig. 1) calculated at $1 \mathrm{GeV} / \mathrm{u}$ with Pauli blocking. As seen from fig. 2, the magnitude of fluctuations associated with $P^{2}$ is a sizable fraction of its mean value $\left\langle P^{2}\right\rangle$ during the interpenetration stage of nuclear collisions. The mean-square deviation exceeds one third of the mean value already at $t=\tau$. However, the magnitude of fluctuations is inversely proportional to the the square-root of the correlation volume (57). Since the value of $V_{\text {corr }}$ is not very well known, our results should be taken as a crude estimate of the magnitude of the local fluctuations in energy-momentum space.

The fact that the fluctuations in $P^{2}$ go to zero at large times rather than take there thermal value is, partially, a consequence of the approximation introduced in the correlator (62). In this correlator the contribution due to the pressure term in the energy-momentum tensor, cf. eq. (45), is neglected. As a result, $P^{2}$ does not contain thermal fluctuations.

\section{Summary and Conclusion}

The relativistic BLE approach provides a useful basis for describing the fluctuation dynamics and for investigating the gross properties of the reaction mechanism of intermediate-energy heavy-ion collisions. However, a direct simulation of 
the BLE is difficult and requires a huge amount of numerical efforts. Therefore, it is reasonable to look for simplified descriptions of the collision process in terms of a few relevant, locally defined, collective variables rather than via the complete phase-space distribution function. In this work we have considered multi-fluid models previously developed at the mean-field level with collisions $[14,15]$. In this multi-fluid approach, the distribution function is decomposed into several equilibrium components. In the present work we have performed the reduction of the relativistic BLE to the stochastic multi-fluid model and have derived transport coefficients, i.e. the interflow friction and diffusion coefficients associated with these local collective variables. This model is particularly suitable for studying the effects of fluctuations during the nonequilibrium interpenetration stage of heavy-ion collisions.

In order to illustrate the formalism and to estimate the magnitude of local fluctuations, we have considered the simple situation of two homogeneous counterstreaming nuclear fluids of normal nuclear density. Our calculations indicate that large local fluctuations occur during the interpenetration stage of nuclear collisions. It would be interesting to investigate the influence of this local fluctuations on the particle production mechanism and on mean-field instabilities which may develop during the interpenetration stage $[3,39]$.

\section{Acknowledgements}

The authors gratefully acknowledge fruitful discussions with members of the theory group at Gesellschaft für Schwerionenforschung. Three of us (S. A, Y. I. and V. R.) highly appreciate the hospitality and support by GSI, where the major part of this work was carried out. One of us (Y. I) also thanks the Tennessee Technological University for the warm hospitality and support during his visit. We are sincerely grateful to T. Ivanova for thoroughly reading and editing the English of the paper.

\section{Appendix: Stochastic Three-Fluid Model}

Here we present results of a reduction of the relativistic BLE to a stochastic three-fluid model. Technically, this reduction is very similar to that described in sect. 3 but is more laborious. We follow the version of the three-fluid model developed in ref. [15]. Since there are transitions of nucleons from the projectile and target fluids into the stopped one within the three-fluid dynamics, the number of nucleons in a separate fluid is not a conserved quantity any more. Consequently, the continuity equations acquire source terms in the r.h.s's

$$
\begin{gathered}
\partial^{\mu} J_{\mu}^{(\alpha)}=S^{(\alpha)}+\delta S^{(\alpha)}, \\
\partial^{\mu} T_{\mu \nu}^{(\alpha)}-g_{\omega} J_{\mu}^{(\alpha)} \omega_{\nu}^{\mu}+g_{\sigma} \rho^{(\alpha)} \partial_{\nu} \sigma=R_{\nu}^{(\alpha)}+\delta R_{\nu}^{(\alpha)}
\end{gathered}
$$

with $\alpha=p, t$ or $s$. The equations for the mean fields are

$$
\left(\square+m_{\sigma}^{2}\right) \sigma+d U / d \sigma=g_{(\sigma)} \sum_{\alpha} \rho^{(\alpha)},
$$




$$
\left(\square+m_{\omega}^{2}\right) \omega_{\mu}=g_{(\omega)} \sum_{\alpha} J_{\mu}^{(\alpha)} .
$$

The average source terms and friction forces are expressed in terms of densities and transport coefficients as follows

$$
\begin{gathered}
S_{\nu}^{(\alpha)}(x)=\left(3 \delta_{\alpha s}-1\right) \bar{B}^{(p t)} \rho^{(p)} \rho^{(t)} / M_{N}^{*} \\
R_{\nu}^{(\alpha)}(x)=\bar{B}^{(p t)} \sum_{\beta}\left(1-\delta_{\beta s}\right)\left(\delta_{\alpha s}-\delta_{\alpha \beta}\right) \rho^{(\bar{\beta})} J_{\nu}^{(\beta)} \\
-\sum_{\beta}\left(1-\delta_{\alpha \beta}\right) \bar{D}^{(\alpha \beta)}\left(J_{\nu}^{(\alpha)} \rho^{(\beta)}-\rho^{(\alpha)} J_{\nu}^{(\beta)}\right) .
\end{gathered}
$$

The correlation functions are determined in terms of the same densities and transport coefficients

$$
\begin{aligned}
\left\langle\delta S^{(\alpha)}(x) \delta S^{\left(\alpha^{\prime}\right)}\left(x^{\prime}\right)\right\rangle= & \delta^{(4)}\left(x-x^{\prime}\right)\left(3 \delta_{\alpha s}-1\right)\left(3 \delta_{\alpha^{\prime} s}-1\right) \bar{B}^{(p t)} \rho^{(p)} \rho^{(t)} / M_{N}^{*} \\
\left\langle\delta S^{(\alpha)}(x) \delta R_{\nu}^{\left(\alpha^{\prime}\right)}\left(x^{\prime}\right)\right\rangle & =\delta^{(4)}\left(x-x^{\prime}\right) \bar{B}^{(p t)} \\
& \times\left\{2 \delta_{\alpha s} \delta_{\alpha^{\prime} s} \sum_{\beta}\left(1-\delta_{\beta s}\right) \rho^{(\bar{\beta})} J_{\nu}^{(\beta)}\right. \\
& \left.+\left(1-3 \delta_{\alpha s}\right)\left(1-\delta_{\alpha^{\prime} s}\right) \rho^{\left(\alpha^{\prime}\right)} J_{\nu}^{\left(\alpha^{\prime}\right)}\right) \\
& \left.\left.-\delta_{\alpha^{\prime} s}\left(1-\delta_{\alpha s}\right)\left(\rho^{(\bar{\alpha})} J_{\nu}^{(\alpha)}\right)+\rho^{(\alpha)} J_{\nu}^{(\bar{\alpha})}\right)\right\} \\
\left\langle\delta R_{\mu}^{(\alpha)}(x) \delta R_{\nu}^{\left(\alpha^{\prime}\right)}\left(x^{\prime}\right)\right\rangle= & \delta^{(4)}\left(x-x^{\prime}\right)\left\{\sum_{\beta \gamma}\left(1-\delta_{\beta \gamma}\right)\left(\delta_{\alpha \gamma}-\delta_{\alpha \beta}\right)\left(\delta_{\alpha^{\prime} \gamma}-\delta_{\alpha^{\prime} \beta}\right)\right. \\
& \times\left[\left(\bar{A}_{\|}^{(\beta \gamma)}-\bar{A}_{\perp}^{(\beta \gamma)}\right)\left(T_{\mu \nu}^{(\beta)} \rho^{(\gamma)}-M_{N}^{*} J_{\mu}^{(\beta)} J_{\nu}^{(\gamma)}\right)\right. \\
+ & \left.\left.\bar{A}_{\perp}^{(\beta \gamma)}\right) g_{\mu \nu} M_{N}^{*}\left(\rho^{(\beta)} \rho^{(\gamma)}-J^{(\beta)} \cdot J^{(\gamma)}\right)\right] \\
+ & \bar{B}^{(p t)}\left[\left(1-\delta_{\alpha s}\right)\left(1-\delta_{\alpha^{\prime} s}\right)\left(\delta_{\alpha^{\prime} \alpha} T_{\mu \nu}^{(\alpha)} \rho^{(\bar{\alpha})}+\delta_{\alpha^{\prime} \bar{\alpha}} M_{N}^{*} J_{\mu}^{(\alpha)} J_{\nu}^{(\bar{\alpha})}\right)\right. \\
& -\delta_{\alpha s}\left(1-\delta_{\alpha^{\prime} s}\right)\left(T_{\mu \nu}^{\left(\alpha^{\prime}\right)} \rho^{\left(\bar{\alpha}^{\prime}\right)}+M_{N}^{*} J_{\nu}^{\left(\alpha^{\prime}\right)} J_{\mu}^{\left(\bar{\alpha}^{\prime}\right)}\right) \\
& -\left(1-\delta_{\alpha s}\right) \delta_{\alpha^{\prime} s}\left(T_{\mu \nu}^{(\alpha)} \rho^{(\bar{\alpha})}+M_{N}^{*} J_{\mu}^{(\alpha)} J_{\nu}^{(\bar{\alpha})}\right) \\
& \left.\left.+\delta_{\alpha s} \delta_{\alpha^{\prime} s} \sum_{\beta}\left(1-\delta_{\beta s}\right)\left(T_{\mu \nu}^{(\beta)} \rho^{(\bar{\beta})}+M_{N}^{*} J_{\mu}^{(\beta)} J_{\nu}^{(\bar{\beta})}\right)\right]\right\} .
\end{aligned}
$$

The transport coefficients are calculated according to the formulae

$$
\begin{gathered}
\bar{B}^{(p t)}=\frac{1}{M_{N}^{*}} \int d^{4} p_{3} d^{4} p_{4} W(1 s 2 s \mid 3 p 4 t) \tilde{F}(3) \tilde{F}(4), \\
\left(\begin{array}{c}
\bar{D}^{(\alpha \beta)} \\
\bar{A}_{\|}^{(\alpha \beta)} \\
\bar{A}_{\perp}^{(\alpha \beta)}
\end{array}\right)=\frac{1}{M_{N}^{*}} \int d^{4} p_{3} d^{4} p_{4} W(1 \alpha 2 \beta \mid 3 \alpha 4 \beta) \tilde{F}(3) \tilde{F}(4)\left(\begin{array}{c}
1-\cos \theta_{c m} \\
\frac{1}{2}\left(1-\cos \theta_{c m}\right)^{2} \\
\frac{1}{6} \sin ^{2} \theta_{c m}
\end{array}\right)
\end{gathered}
$$

at an intermediate value

$$
\bar{s}^{(\alpha \beta)}=M_{N}^{* 2}\left\{\left(u^{(\alpha)}+u^{(\beta)}\right)^{2}+2\left(u^{(\alpha)} \cdot u^{(\beta)}\right)\left[\left(n^{(\alpha)} / \rho^{(\alpha)}\right)\left(n^{(\beta)} / \rho^{(\beta)}\right)-1\right]\right\}
$$

of the $s_{12}=\left(p_{1}+p_{2}\right)^{2}$ argument. 


\section{References}

[1] H.-T. Elze, M. Gyulassy, D. Vasak, H. Heinz, H. Stöcker and W. Greiner, Mod. Phys. Lett. A2 (1987) 451

[2] Y. B. Ivanov, Nucl. Phys. A474 (1987) 669

[3] Y. B. Ivanov, in "Relativistic Heavy-Ion Physics", eds. L. P. Csernai and D. Strottman, Int. Rev. of Nucl. Phys., vol. 6, p. 426-594 (World Scientific, Singapore, 1991)

[4] W. Botermans and R. Malfliet, Phys. Lett. B215 (1988) 617

[5] Q. Li, J. Q. Wu and C. M. Ko, Phys. Rev. C39 (1989) 849

[6] M. Cubero, M. Schönhofen, B. L. Friman and W. Nörenberg, Nucl. Phys. A519 (1990) 345c

[7] M. Schönhofen, PhD thesis (TH Darmstadt, 1990); M. Schönhofen, M. Cubero, B. Friman and W. Nörenberg, Nucl. Phys. A (submitted)

[8] M. Cubero, PhD thesis (TH Darmstadt, 1990), GSI-Report-90-17; M. Cubero, M. Schönhofen, B. Friman and W. Nörenberg, to be published

[9] S.-J. Wang, B.-A. Li, W. Bauer and J. Randrup, Ann. of Phys. 209 (1991) 251

[10] P. Danielewicz, Ann. Phys. 197 (1990) 154

[11] C. M. Ko, Q. Li and R. Wong, Phys. Rev. Lett. 59 (1987) 1084;

C. M. Ko and Q. Li, Phys. Rev. C37 (1988) 2270;

C. M. Ko, Nucl. Phys. A495 (1989) 321c

[12] B. Blättel, V. Koch, W. Cassing and U. Mosel, Phys. Rev. C38 (1988) 1767;

B. Blättel, V. Koch, K. Weber, W. Cassing and U. Mosel, Nucl. Phys. A495 (1989) 281c;

V. Koch, B. Blättel, W. Cassing and U. Mosel, Phys. Lett. B236 (1990) 135;

V. Koch, B. Blättel, W. Cassing, U. Mosel and K. Weber, Phys. Lett. B241 (1990) 174;

K. Weber, B. Blättel, V. Koch, A. Lang, W. Cassing and U. Mosel, Nucl. Phys. A515 (1990) 747

[13] W. Cassing and U. Mosel, Progr. Part. and Nucl. Phys. 25 (1990) 1

[14] Y.B. Ivanov, V.N. Russkikh, M. Schönhofen, M. Cubero, B.L. Friman and W. Nörenberg, Z. Phys. A340 (1991) 385 
[15] Y.B. Ivanov, V.N. Russkikh, M. Schönhofen, M. Cubero, B.L. Friman and W. Nörenberg, Proc. of Int. Workshop on Gross Properties of Nuclei and Nuclear Excitations $X X$, Hirschegg, Austria, January 1992, Ed. by H. Feldmeier, Darmstadt 1990, p. 239

[16] W. Bauer, G. F. Bertsch and S. Das Gupta, Phys. Rev. Lett. 58 (1987) 963

[17] S. Ayik and C. Gregoire, Phys. Lett. B212 (1988) 269 and Nucl. Phys. A513 (1990) 187

[18] E. Suraud, S. Ayik, M. Belkacem and J. Stryjewski, Nucl. Phys. A542 (1992) 141

[19] P. G. Reinhard, E. Suraud and S. Ayik, Ann. Phys. 223 (1992) 2r 4;

P. G. Reinhard and E. Suraud, Ann. Phys. (N.Y.) 216 (1992) 98

[20] J. Randrup and B. Remaud, Nucl. Phys. A514 (1990) 339

[21] Ph. Chomaz, G. F. Burgio and J. Randrup, Phys. Lett. B254 (1991) 340; G. F. Burgio, Ph. Chomaz and J. Randrup, Nucl. Phys. A529 (1991) 154

[22] S. Ayik, Phys. Lett. B265 (1991) 47

[23] H. Feldmeier, M. Schönhofen and M. Cubero, Nucl. Phys. A495 (1989) $337 \mathrm{c}$

[24] J. D. Walecka, Ann. of Phys. 83 (1974) 491

[25] B. D. Serot and J. D. Walecka, The Relativistic Nuclear Many-Body Problem, Adv. Nucl. Sci. 16 (1986) 1

[26] A. A. Amsden, A. S. Goldhaber, F. H. Harlow and J. R. Nix, Phys. Rev. C17 (1978) 2080

[27] R. B. Clare and D. Strottman, Phys. Rep. 141 (1986) 178

[28] I. N. Mishustin, V. N. Russkikh and L. M. Satarov, Nucl. Phys. A494 (1989) 595 and in "Relativistic Heavy-Ion Physics", eds. L. P. Csernai and D. Strottman, Int. Rev. of Nucl. Phys., vol. 5 (World Scientific, Singapore, 1991) p. 180

[29] L. M. Satarov, Yad. Fiz. [Sov. J. Nucl. Phys.] 52 (1990) 412

[30] H. W. Barz, B. Kämpfer, L. P. Csernai and B. Lukacs, Nucl. Phys. A465 (1987) 742; H. W. Barz and B. Kämpfer, Phys. Lett. 206B (1988) 399

[31] L. P. Csernai, I. Lovas, J. A. Maruhn, A. Rosenhauer, J. Zimanyi, and W. Greiner, Phys. Rev. C26 (1982) 149; A. Rosenhauer, L. P. Csernai, J. A. Maruhn, and W. Greiner, Phys. Scr. 30 (1984) 45

[32] A. Rosenhauer, J. A. Maruhn, and W. Greiner, Z. Phys. A326 (1987) 213 
[33] M. Schönhofen, M. Cubero, M. Gering, M. Sambataro, H. Feldmeier and W. Nörenberg, Nucl. Phys. A504 (1989) 875

[34] H. Risken, The Fokker-Planck Equation (Springer-Verlag Berlin Heidelberg, 1989)

[35] Yu. B. Ivanov, I. N. Mishustin and L. M. Satarov, Nucl. Phys. A433 (1985) 713; Yu. B.Ivanov and L. M. Satarov, Nucl. Phys. A446 (1985) 727

[36] J. Cugnon, Phys. Rev. C22 (1980) 1885

[37] M. Belkacem, E. Suraud and S. Ayik, Phys. Rev. C47 (1993) R16

[38] D. L' Hôte and J. Cugnon, in "Relativistic Heavy-Ion Physics", eds. L. P. Csernai and D. Strottman, Int. Rev. of Nucl. Phys., vol. 5 (World Scientific, Singapore, 1991) p. 37

[39] Yu. B. Ivanov, Nucl. Phys. A474 (1987) 693 


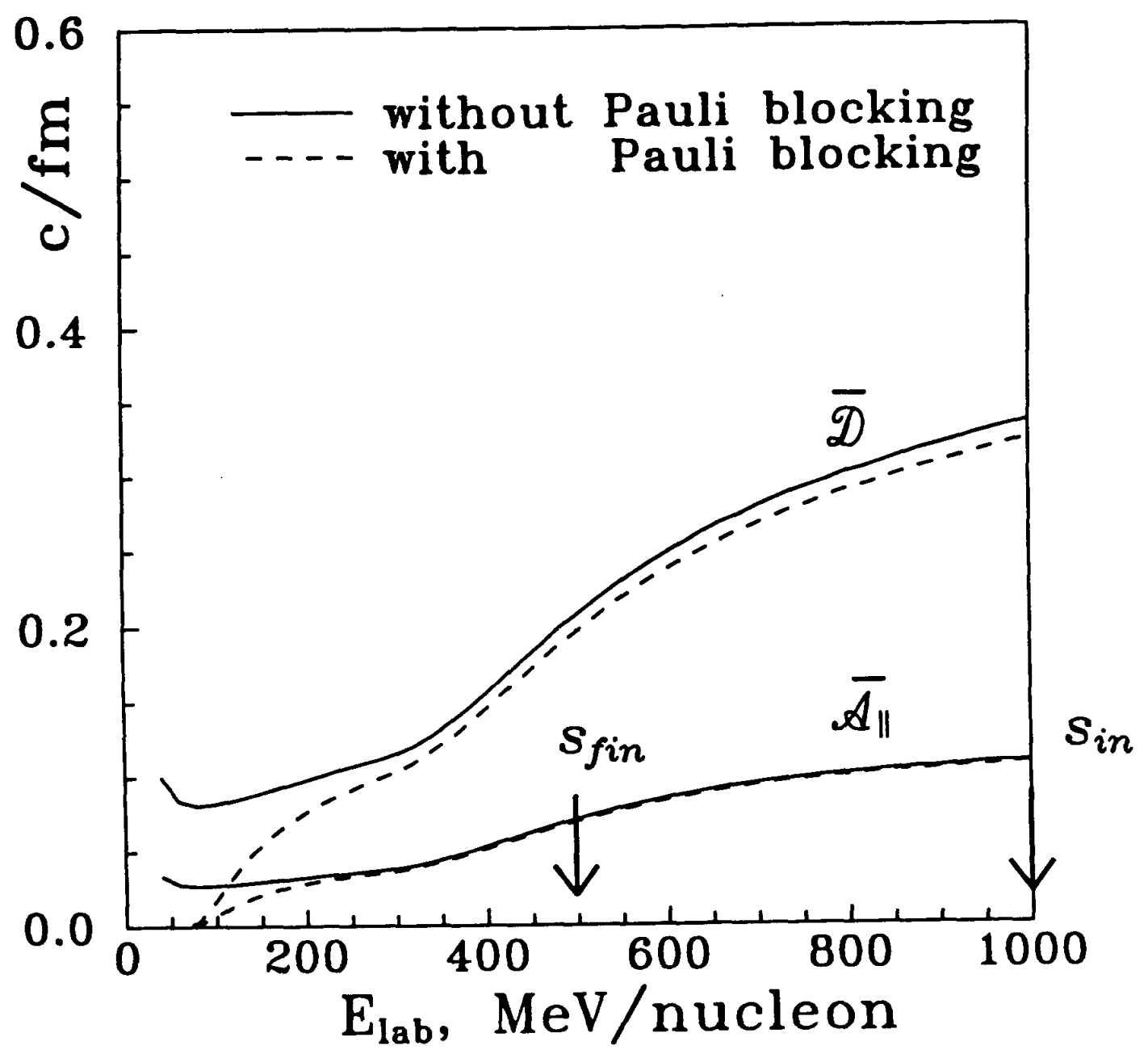

Fig. 1. Energy dependence of the $\overline{\mathcal{D}}$ and $\overline{\mathcal{A}}_{\|}$transport coefficients calculated with and without Pauli blocking. 


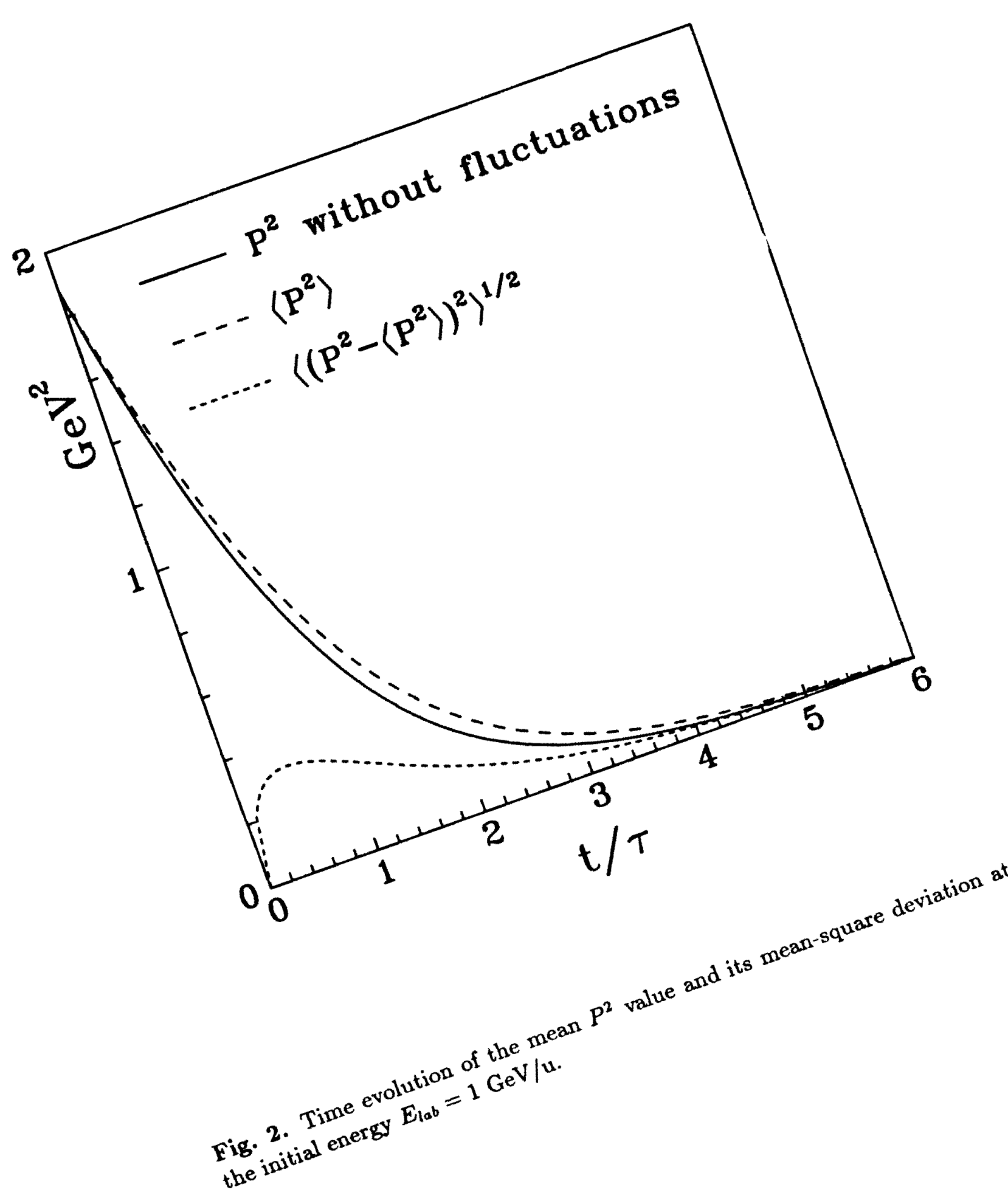


